\title{
The role of spinal fusion in surgery for lumbar spinal stenosis: a review
}

\section{Anthony J. Caputy, M.D., Caple A. Spence, M.D., Ghassan K. Bejjani, M.D., and Alfred J. Luessenhop, M.D.}

Department of Neurosurgery, The George Washington University Medical Center, and Department of Neurosurgery, Georgetown University, Washington, D.C.

The authors undertook a review of the literature and analysis of the local surgical experience for lumbar stenosis to define the role of simultaneous arthrodesis in the treatment of patients undergoing decompression for spinal stenosis. The restrained use of spinal fusion is recommended in spinal stenosis surgery because of the coexisting medical problems in the elderly patient population and the higher associated complication rate with spinal fusion and instrumentation. A spinal fusion is recommended when decompression is performed in an area of segmental instability as manifested by gross movement on flexion-extension radiographs; when the decompression coincides with an area of degenerative instability, as with scoliosis or spondylolisthesis; or when the decompression creates an iatrogenic instability by the disruption of the posterior elements. The use of spine instrumentation as an adjunct to fusion is recommended when an area of degenerative instability shows evident gross instability or has had additional destabilizing procedures, such as a discectomy or a facetectomy. Spinal fusion is not recommended for a routine decompressive laminectomy for lumbar stenosis or in the case of stable degenerative deformities. New fusion techniques may improve the outcome and decrease the morbidity associated with contemporary methods of spinal fusion and instrumentation.

\section{Key Words * laminectomy * lumbar spine * spondylolisthesis * scoliosis * spinal fusion * instrumentation}

Lumbar spinal stenosis is a well-established clinical syndrome for which surgical decompression has a proven benefit. However, the role of spinal fusion in the treatment of patients undergoing lumbar decompression for spinal stenosis has not been well elucidated. Advances in diagnostic imaging, surgical techniques, and the explosion of devices for internal fixation in the past 15 years have led to the expansion of the indications for spinal fusion. One of the most controversial areas in the surgical treatment of degenerative lumbar spine disease concerns the implementation of spinal fusion in the treatment of lumbar stenosis. This paper will review the role of simultaneous arthrodesis in the treatment of patients undergoing lumbar decompression surgery for spinal stenosis and will address preoperative selection criteria.

The first clinical report of a spinal fusion was in 1911 by Albee[2] who used tibia graft implanted in the 
spinous processes to treat Pott's disease. In 1917 Hibbs[27] used a spinal fusion technique to treat a progressive deformity in scoliosis. Since that time, surgeons have used a variety of anterior, posterior, or posterolateral approaches for spine fusions. The applications have broadened and fusion has been used in the management of spinal fractures, primary and metastatic tumors, spinal deformities, disc herniations,[29] and in the treatment of degenerative spine diseases such as scoliosis, spondylolisthesis, and spinal stenosis. Devices for the internal fixation of the spine have been fabricated and refined to augment spinal fusion techniques. Since the first initial report by Lang[39] in 1910 of the use of steel rods to support a spondylitic spine, a wide variety of devices have been developed to provide internal support for the spine in an attempt to restore the spinal architecture, assist in the initial postoperative stabilization of the spine, and to promote an increased rate of fusion consolidation.

\section{PATHOPHYSIOLOGY OF DEGENERATIVE LUMBAR SPINE DISEASE}

Yong-Hing and Kirkaldy-Willis[66] outlined the pathogenesis and natural history of degenerative lumbar spine disease by meticulously examining at autopsy a large number of spines in different stages of degeneration. They defined, at each level of the spine, a "three-joint complex" consisting of the intervertebral disc and the two posterior zygoapophyseal joints. These three joints function as one unit so that a pathological change in one joint will affect the other joints at that level and ultimately the adjacent spinal levels. The resulting interaction may give rise to multilevel degenerative conditions such as lumbar spondylosis, stenosis, or spondylolisthesis. The degenerative process involving the lumbar spine was separated into three stages: 1) dysfunction, 2) instability, and 3) stabilization. The dysfunction stage is characterized by nuclear degeneration, annular tears, and facet arthropathy. The stage of instability follows with the essential pathological finding of abnormal movement in the spinal motion segment as exhibited by laxity of the facets and ligaments, reduced disc space height, and increased motion. A degenerative spondylolisthesis may develop during this stage. As the stage of instability is resolved, an attempt to restabilize the spine ensues in the stabilization phase. This third phase shows the effect of severe degeneration with the characteristic formation of osteophytes, facet hypertrophy, and disc desiccation with increased intradiscal collagen. There is reduced movement and the fixed nerve roots become entrapped with the resultant clinical symptoms of spinal stenosis.[66]

It was thought by Yong-Hing and Kirkaldy-Willis[66] that if decompressive surgery for symptoms of spinal stenosis should become necessary during the stage of "instability," then a simultaneous fusion should be performed to stabilize the motion segment. However, they caution that there is a correlative risk of producing an unstable condition at the level above a successful fusion. They further conclude that in the last stage of "stabilization," decompression alone for spinal stenosis should be sufficient to alleviate the symptoms without fusion.

\section{SPINAL STENOSIS: NATURAL HISTORY AND MANAGEMENT}

The clinical definition of degenerative lumbar stenosis is the production of radicular symptoms or symptomatic neurogenic claudication as the result of structural changes produced by a combination of laminar thickening, ligamentous hypertrophy, facet hypertrophy, and annular bulging, with or without spondylolisthesis or scoliotic angulation, which leads to the crowding of adjacent nerve roots or the cauda equina. The natural nonoperative history of patients with symptomatic spinal stenosis and myelography-proven disease has shown that in $85 \%$ of these patients the pain was the same or worse over a long-term follow-up period.[32]

The results of surgical decompression for spinal stenosis found in the literature have been shown to be 
better than those of long-term nonoperative management. Since the early reports by Verbiest,[63] there have been a series of articles describing the outcomes of various surgical procedures used to treat lumbar stenosis.[7,11,17,19,24,25,28,33,34,42,49,59,61,63,64,68] The most common surgery involves a laminectomy with a partial medial facetectomy. However, the reported surgeries range from a limited decompression via laminotomy to a more radical removal of the posterior elements combined with instrumentation and fusion. Poor surgical results have often been ascribed to persistent levels of instability or increased instability caused by decompression.[7,28,31,35,42,66] As a result, simultaneous fusions have been advocated. However, the association of improved clinical outcome with instrumentation and fusion has not been firmly established, and a high rate of complications has also been associated with the concomitant use of spinal fusion or spinal fusion with instrumentation. Attempts must be made to define those patients who are likely to benefit from the fusion and in whom the increased risk of fusion becomes justified by an expected improved outcome.

\section{Surgical Decompression for Lumbar Stenosis}

Studies have reported satisfactory long-term outcomes in lumbar decompressive surgeries for spinal stenosis without fusion.[18,25,26,31,32,34,42,47,49,58,59,64]

Surin, et al.,[59] in a 1982 article, reviewed the results of decompressive laminectomies for spinal stenosis in 22 patients with a mean age of 64 years. No fusions were performed in this group of patients. Two patients developed spondylolisthesis, and two patients experienced worsening of existing spondylolisthesis. Three of these four patients were considered to have had satisfactory results with only symptoms of slight backache. The authors concluded that patients with an existing spondylolisthesis tended to show progressive spinal instability. However, they could not attribute any of the postoperative symptoms to this finding and therefore did not recommend fusion as an adjunct to decompression for lumbar stenosis.

In a 1993 study, Silvers, et al.,[54] performed a retrospective analysis of 244 patients undergoing decompressive laminectomy for lumbar stenosis (mean age 65 years). Fifty-two patients had spondylolisthesis, and $17 \%$ of the patients underwent discectomy in addition to the lumbar decompression for stenosis. The authors reported a success rate of 93\%. Only two patients underwent a subsequent spinal fusion, and that was at the time of reoperation in the early postoperative period to perform a more extensive decompression. The mean age of the group of patients with existing spondylolisthesis was 67 years. Six of 52 patients in this group underwent repeated surgeries for renewed neurological symptoms. No fusions were performed at the time of the reoperation, and five of these six patients were considered to have achieved a successful outcome. This has been interpreted by the authors as obviating the need for a fusion. However, this may also be indicative of a postlaminectomy bone regrowth from instability as described by others.[6-8,50,63,66]

In a local experience, Caputy and Luessenhop[7] examined a series of 100 patients (mean age 67 years) who underwent surgery for degenerative lumbar stenosis. They were reviewed over a 10- to 15-year follow-up period, with failures accumulating over many years. Total laminectomies and partial facetectomies were performed in $85 \%$ in the cases, with unilateral decompression being undertaken in the remaining $15 \%$. There were no instances of simultaneous fusions. The surgeries in 26 patients were considered failures: 16 as the result of renewed radicular or cauda equina involvement and 10 because of persisting or worsening back pain. There was a disproportionately higher incidence of coexisting spondylolisthesis among the surgical failures (12 of 26 patients). The tendency for recurrence of 
spondylolisthetic stenosis following decompressive laminectomy led to the recommendation that concurrent fusion be performed with the decompression at a level of an existing spondylolisthesis.

\section{INSTABILITY AND FUSION}

A clear definition of what constitutes spinal instability or what conditions promote spinal instability is essential to predict what portion of the patient population undergoing lumbar decompression for stenosis would benefit most from a simultaneous fusion. The concordant topics of degenerative spondylolisthesis, degenerative scoliosis, and decompressions that involve a significant multilevel decompression or the disruption of the posterior elements will be addressed in the following discussion.

Measurements of spinal instability in patients with spondylolisthesis may be determined on flexion-extension x-ray films. Objective or "gross instability" has been defined as greater than $4 \mathrm{~mm}$ of translation or 10š of angular change on standing lateral flexion-extension x-ray films.[21]

Frymoyer[16] has attempted to categorize degenerative segmental instabilities into either primary or secondary instabilities. The primary instabilities arise de novo without prior surgical intervention and include rotational, translational, spondylolisthetic, and scoliotic progressive degenerative changes. The secondary segmental instabilities occur following surgery and include procedures involving disc excisions, decompressive laminectomies, and fusions.

Hanley's[20] review has categorized unstable subsets in lumbar spinal stenosis in an attempt to define the patients likely to benefit from fusion. Hanley proposes an "absolute stenosis group" in whom he believes the disease is stable and in whom decompressive procedures alone will relieve the symptoms of spinal stenosis. This group includes patients with lateral recess stenosis or multisegment ankylosing stenosis. The "unstable stenosis group" includes patients with degenerative spondylolisthesis and degenerative scoliosis. Spinal fusion at the time of decompressive surgery for symptoms of spinal stenosis in these patients is often recommended. In a review of indications for lumbar fusion in adults, Esses and Huler[12] recommended simultaneous fusion when decompression was performed in the presence of spondylolisthesis, scoliosis, or discectomy. Others have also reported similar conclusions.[5,24,43,47,51,56,68]

Segmental instrumentation and fusion have been proposed for those patients undergoing decompression at a level of degenerative spondylolisthesis, as well as those who exhibit gross instability preoperatively at the level of the spondylolisthesis. A noninstrumented posterior fusion was advocated for the group of patients without evidence of "gross instability" at a decompressed level of spondylolisthetic stenosis.[20,35,53]

\section{Degenerative Spondylolisthesis}

The surgical management of spinal stenosis with associated degenerative spondylolisthesis at the same level has involved decompression alone, a combination of decompression and fusion, or a delayed fusion. Sonntag and Marciano[56] reviewed 115 patients who underwent decompressive laminectomy for spinal stenosis. Spines in seven patients became unstable and required subsequent fusion. In addition to the 20 patients with existing spondylolisthesis, four required subsequent fusion. The authors concluded that there was no conclusive evidence that fusion would improve the outcome in patients undergoing decompressive laminectomy at the site of a Grade I spondylolisthesis. However, fusion should be considered in those patients with mechanical back pain and associated Grade II or higher spondylolisthesis. 
Herkowitz and Kurz[24] undertook a prospective study of 50 patients with spinal stenosis and associated spondylolisthesis. Half of these patients underwent decompression only, whereas the remaining half underwent decompression with intertransverse fusion. Good-to-excellent outcomes were reported in $96 \%$ of those undergoing fusion, whereas only $44 \%$ of the nonfusion group had similar outcomes. The difference in outcome was thought to be due to the progression of the spondylolisthesis in the nonfusion group. However, it was additionally noted that there was a $36 \%$ pseudarthrosis rate in the fusion group. This incidence of pseudarthrosis had no effect on the outcome; all of those patients with pseudarthrosis reported good or excellent outcomes. It was speculated that a solid fusion on one side of the operative area or the development of a fibrous union would provide sufficient support to prevent the progression of the spondylolisthesis.

Similar results were found in another prospective study by Bridwell, et al.,[5] in which 44 patients with degenerative spondylolisthesis and stenosis were divided into three groups. The first group underwent decompressive laminectomy alone; the second group, decompression and intertransverse fusion; and the third group, decompression and fusion with pedicle screw instrumentation. This finding of inferred stability of the spondylolisthetic motion segment--established by a lack of progression of the spondylolisthesis--has been associated with a better prognosis and is more frequent in the fusion or fusion with instrumentation groups.[5,24,31,33,51,67] Other authors have found more favorable results in patients undergoing simultaneous fusion at the time of decompression in areas of spondylolisthesis.[13,24,28,35,43,53]

The progression of a spondylolisthesis with or without fusion has been noted. Studies have reported that patients undergoing fusion had a better clinical outcome irrespective of the degree of progression of the spondylolisthesis.[17,24,64] Others, however, have found no relationship between the postoperative progression of spondylolithesis and the clinical outcome.[13,14,26,33,40,48,59]

Lee[40] studied instability following spinal decompression and concluded that the routine use of fusion in conjunction with decompression for stenosis was not indicated. This study reviewed a small group of 27 patients with a mean age of 49.4 years. Here the incidence of a new spondylolisthesis following decompression was $3.7 \%$, and $100 \%$ of the patients with existing spondylolisthesis progressed. The fusion did not appear to prevent the development of new spondylolisthesis, and it did not retard the progression of that already in existence.

Lehmann, et al.,[41] analyzed the long-term results in patients undergoing lumbar fusion and found that, although $85 \%$ of the patients were satisfied with their outcomes 3 years after surgery, radiographic evidence in $50 \%$ of those patients showed segmental instability or stenosis. This finding emphasized the fact that radiographic changes did not correlate with the clinical symptoms. The authors also found that patients undergoing fusion for spondylosis or degenerative spondylolisthesis had a better outcome than those undergoing fusion for other reasons, such as trauma, infection, or neoplasm.

Herron and Trippi[26] reported 24 patients (mean age 67 years) with L4-5 spondylolisthesis who underwent decompressive laminectomy and partial facetectomy without fusion. The authors concluded that the routine use of a spinal fusion in degenerative spondylolisthesis is not necessary. They added that spinal fusion may be appropriate if there is objective evidence of spinal instability on preoperative flexion-extension x-ray films or if the integrity of the pars interarticularis was violated during surgery.

In their 1988 series of decompressive laminectomies, Hopp and Tsou[28] reported a 17\% reoperation rate for instability related to spondylolisthesis. They found that preoperative indicators of potential 
instability included the presence of a degenerative disc with traction spurs, diminished disc height, and the presence of spondylolisthesis, scoliosis, or asymmetry in the narrowed disc space. They also found that those procedures involving a total facetectomy and/or destabilization of the pars interarticularis resulted in an increased risk of instability. Fusion was recommended when these preoperative risk factors were present. [16,28]

\section{Age as an Indicator for Fusion}

Age was seen to be a factor in the need for spinal fusion in patients undergoing decompression for stenosis. Several authors have concluded that the outcome for spinal decompression for stenosis improved in patients younger than 60 or 65 years if a simultaneous fusion was performed at the initial decompression. The use of fusion was not shown to be of significant benefit in older patients.[3,31,40,51,64] Alexander, et al.,[3] concluded that the indications for fusion should be diminished in patients older than 50 years and should be rare after 60 years. They attributed this to the increased osteoarthritic stability with age. However, they did favor fusion in older patients if spinal instability was demonstrated.

Wiltse, et al.,[64] did not routinely perform spinal fusion in patients undergoing decompression for lumbar stenosis except for patients: 1) younger than 60 years of age with spondylolisthesis at the decompressed level who underwent bilateral facetectomy; 2) younger than 55 years of age if the decompression was at the level of spondylolisthesis with intact facet joints; and 3) younger than 50 years of age with isthmic spondylolisthesis.

The data from reports on decompressive surgery at areas of spondylolisthetic stenosis have shown that attempts to stabilize an area of spondylolisthetic stenosis with fusion have not prevented the radiographic progression of the spondylolisthesis. In many instances, an improved clinical outcome has been more consistently shown in patients undergoing simultaneous fusion following decompression at the level of an existing spondylolisthesis.

\section{Degenerative Scoliosis}

Degenerative scoliosis in the adult population is associated with disc asymmetry, facet joint hypertrophy, and rotational angulation. These pathological findings may reduce the spinal canal to such a significant degree as to cause symptomatic spinal stenosis that manifests in back pain, radicular pain, or neurogenic claudication. Symptoms that become refractory to conservative management often require decompression and decompression procedures alone have been proposed by some authors.[46,52] However, these patients with degenerative scoliosis are frequently osteoporotic and a decompression alone often results in surgical failures due to progressive back pain and radicular symptoms.[21]

San Martino, et al.,[52] evaluated 20 patients with radicular symptoms caused by degenerative scoliosis. They underwent decompression along the concave portion of the scoliotic curve without fusion. All 20 patients experienced pain relief. No symptoms recurred and no patient required further surgery during the follow-up period of 1 to 4 years. The authors emphasized the importance of the preservation of the facets and the pars interarticularis. It has been suggested that limited decompression of a single symptomatic nerve root in patients without back pain has produced successful outcomes without a concomitant fusion.[20] Some authors have recommended more aggressive surgical approaches that include decompression with correction of deformity, instrumentation, and fusion.[21,38,55]

\section{Multilevel Laminotomies}


As an alternative to multilevel decompressive laminectomies, some authors have proposed a more limited decompression for radicular symptoms and lateral recess stenosis.[4,18] Aryanpur and Ducker[4] proposed that pathological compression involved in lumbar stenosis is a progressive process with subsequent incremental compressive changes exhibiting a more profound effect on clinical symptoms. They believed that laminotomy was sufficient to relieve the symptoms of lateral recess stenosis. The benefit of this approach was a reduction in the perioperative morbidity and a lessening of the destabilizing effects inherent in a wide laminectomy. Thirty-two patients with a mean age of 66 years were presented. There were no morbidities and no mortalities, but one patient was considered a failure and was found to have had progression of existing Grade I spondylolisthesis at the operative level. They recommended that patients with spinal instability undergo a simultaneous fusion with decompression.

In a prospective study by Grob, et al.,[18] 45 patients were divided into three groups to compare the results of: 1) decompressive laminectomy alone, 2) decompressive laminectomy with fusion and instrumentation of the most stenotic segment, and 3) decompression and fusion with instrumentation of all decompressed segments. Patients with preoperative spinal instability were not included. The surgical procedure involved limited decompressive laminotomies of the lateral recess at the symptomatic levels. Discectomies were performed in 20 patients. It was concluded that in the absence of preoperative evidence of instability, fusion is not indicated in patients undergoing a limited decompressive laminectomy with preservation of the posterior elements. In addition, there was a low likelihood that symptomatic segmental instability would develop. They also concluded that in any situation involving limited decompression with laminotomy, the use of spinal instrumentation and fusion increased the operative risks because of increased operative time, increased blood loss, and the complications associated with the instrumentation.[18] The complications identified with the fusion procedure were ileus, increased blood transfusion rate, increased wound infections, deep vein thrombosis, neural injury, pseudarthrosis, graft site pain, and pain localized to the adjacent nonfused motion segment.[10,15,36,39,62]

\section{Posterior Element Disruption}

Biomechanical and clinical studies have shown that a disruption of $50 \%$ of the facets bilaterally or the unilateral removal of one facet joint may compromise the mechanical support of the spine and may produce an unstable motion segment.[1,20-22,26,28,31,35,40,51,52,58,64] Outcomes have been shown to be appreciably worse in younger patients, patients undergoing a simultaneous disc excision, or in patients with inherently unstable pathological conditions such as spondylolisthesis or scoliosis.[16,20,31,40,56]

Hazlett and Kinnard[22] reported 33 patients undergoing decompressive laminectomies in whom at least one entire facet was removed. Four patients demonstrated segmental instability on postoperative x-ray films. The clinical outcome, however, was good in two of the four patients. Lee[40] reviewed 27 patients, with a mean age of 49.4 years, undergoing a radical decompression with posterior element disruption and iatrogenic segmental instability. It was found that only $3.7 \%$ developed a new spondylolisthesis; however, $100 \%$ of the patients with an existing spondylolithesis showed progression. Those patients older than 60 years of age, with decreased disc space height and osteophytes, are generally less likely to require fusion.

Lehto and Honkanen[42] also concluded that patients undergoing single-level decompression for spinal stenosis did not require fusion if the posterior elements were preserved. Multilevel decompressions or 
decompressions at a level of spondylolisthesis had improved outcomes with concomitant fusion.

\section{FUSION WITH INSTRUMENTATION}

The use of spinal instrumentation to augment fusion techniques has been found by some authors to produce a higher fusion rate.[19,45,53,67,68]

Mardjetko, et al.,[45] reviewed 55 papers in a metaanalysis involving 889 patients with degenerative spondylolisthesis and concluded that spinal fusion procedures improved outcomes in the surgical management of degenerative spondylolithesis and that the use of adjunctive spinal instrumentation enhanced the fusion rate. This has been found by other authors.[35,37,45,53-55,67,68] A retrospective study by Yuan, et al.,[67] examining a cohort of patients undergoing pedicle screw fixation for degenerative spondylolisthesis, found that when compared with a group of patients undergoing decompressive surgery with fusion for spondylolisthesis without pedicle screw fixation, the group of patients with simultaneous pedicle screw fixation had better maintenance of spinal alignment, a more rapid fusion mass consolidation, and significantly higher fusion rates ( $89.1 \%$ vs. $70.4 \%$ for the noninstrumented fusion group). It was concluded that, in addition to the fusion enhancement, the patients in the pedicle screw fusion group had a better clinical outcome with less pain, improved function, and greater neurological recovery than the noninstrumented group. They found that device-related complications were infrequent and were not significantly different from complications encountered in the noninstrumented fusion group. Other authors, however, found a higher associated complication rate with the use of spinal instrumentation.[15,18,36]

At the 1996 meeting of the North American Spine Society, Fischgrund, et al., (unpublished data) examined the use of instrumentation to augment fusion techniques in patients with spinal stenosis who had undergone decompression at the level of spondylolisthesis. The authors found a higher fusion success rate as measured by radiographic criteria but discovered no significant improvement in the clinical outcome. They presented 76 patients in a prospective, randomized study who had undergone decompression for spinal stenosis at the level of spondylolisthesis; the patients also received a simultaneous intertransverse fusion alone or simultaneous intertransverse fusion with posterior segmental instrumentation. There was no significant difference in the clinical outcome, although there was a significantly higher rate of fusion success in the group undergoing simultaneous instrumentation. Successful fusion was achieved in $82 \%$ of the instrumented cases versus $45 \%$ of the noninstrumented cases.

\section{BONE REGROWTH AFTER DECOMPRESSIVE LAMINECTOMY}

Bone regrowth at the surgical site is part of the natural long-term postoperative course in patients who have undergone decompressive laminectomy for lumbar stenosis. Some authors have implicated excessive bone regrowth in the recurrence of neurological symptoms. [6-8,50,63] Studies have indicated that there is a progressive increase in bone regrowth as the follow-up period lengthens. Bone regrowth was accelerated in areas of postoperative spinal instability, and this regrowth has led to a recurrence of neurological symptoms (Fig. 1). Other factors associated with increased bone regrowth were more than three spinal levels of decompression and spinal segments adjacent to a previous fusion site. $[6,8,17,41,49,66]$ The increased bone growth may be inherently part of the primary pathological manifestation of lumbar stenosis: it has been noted that the more extensive the pathological findings on the presurgical myelography, then the more extensive the initial decompression, the greater the subsequent acceleration of bone regrowth, and the earlier the recurrence of symptoms. $[8,66]$ 


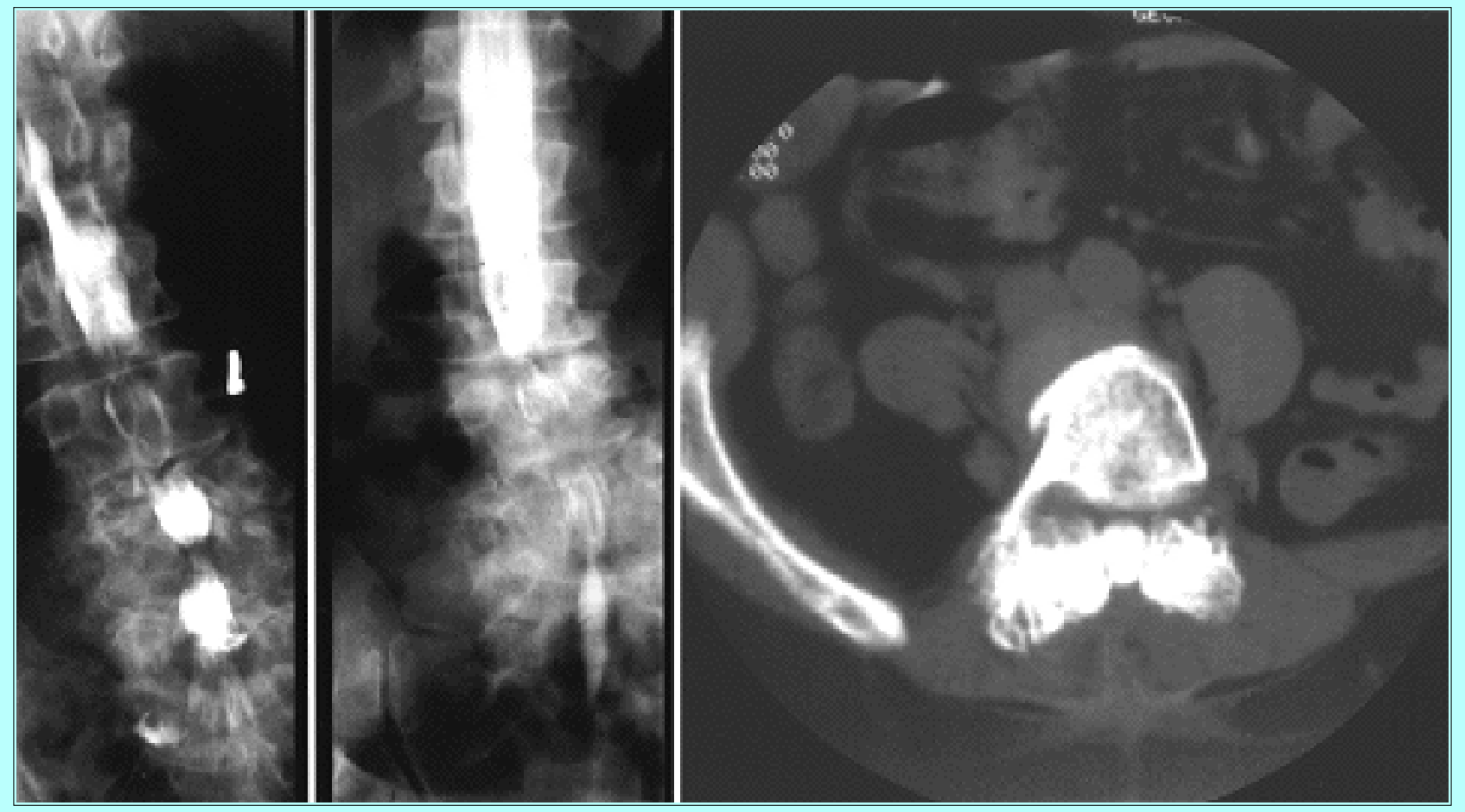

Fig. 1. Left: Anteroposterior myelogram obtained in a 77-year-old woman with symptoms of neurogenic claudication. Radiographs showed severe degenerative spine disease with multiple cascading levels of spondylolisthetic changes and degenerative scoliosis. A multilevel decompressive laminectomy was performed, and the patient remained free of lower-extremity symptoms, with moderate back pain, for 3 years until she developed renewed symptoms of neurogenic claudication. Center: Repeated myelogram obtained 3 years after surgery showed bone regrowth at the previously decompressed area with marked canal stenosis. Right: Computerized tomography scan corresponding to the myelogram in center showing the hypertrophic bone regrowth in the area of degenerative instability.

In the local long-term study of decompressive surgery for spinal stenosis, four patients were evaluated who had new stenosis above a previous spinal fusion site. These patients underwent decompression of the stenotic segments above the existing fusion without the fusion being extended to the newly decompressed area. Although these patients were included in the successful fusion group, it was believed that the previous fusion had accelerated the degenerative process at the adjacent levels.[7] An example of postfusion stenosis is presented in Fig. 2. 


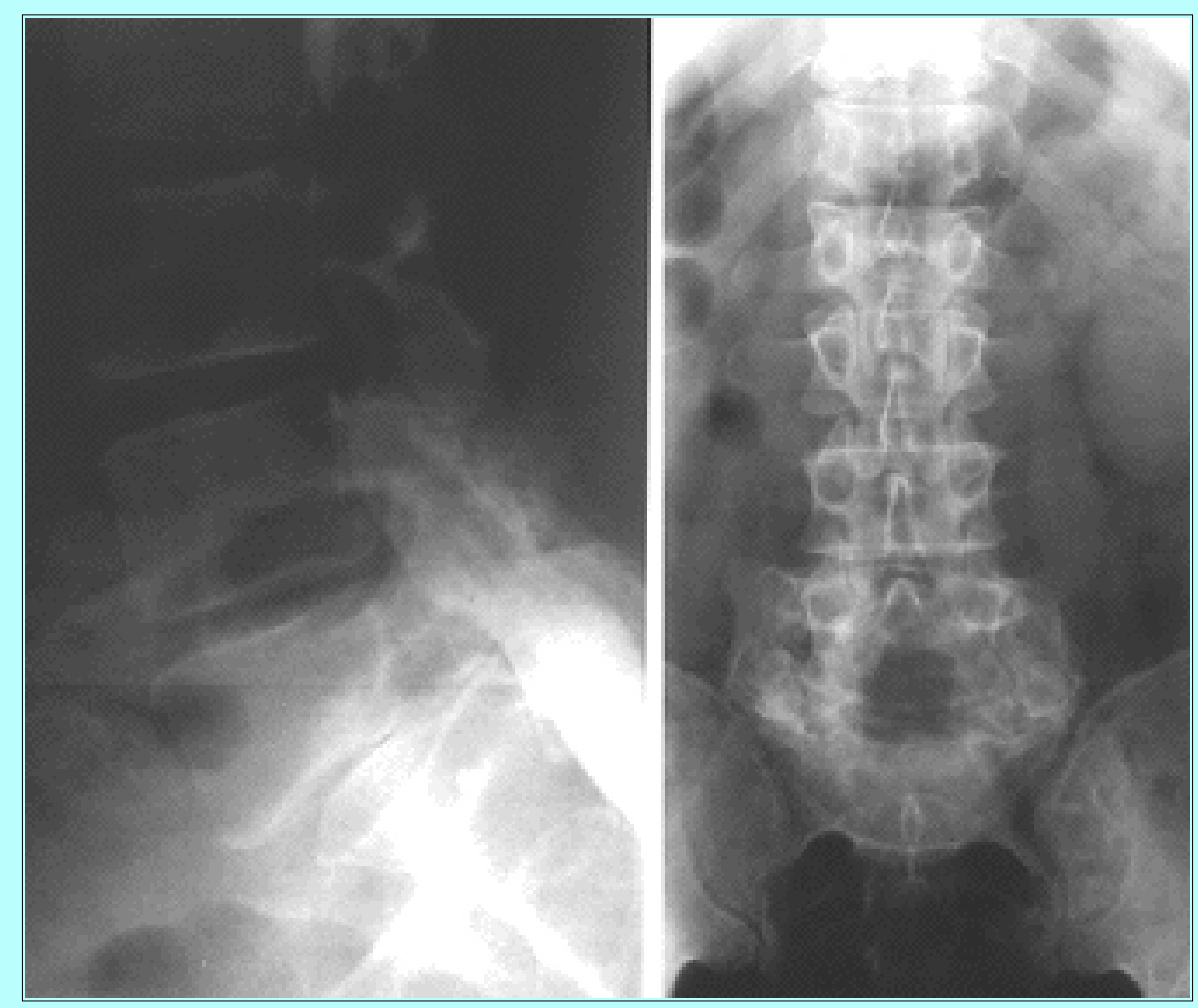

Fig. 2. Left: Postoperative anteroposterior radiograph obtained in a 50-year-old man who underwent decompressive laminectomy with intertransverse iliac crest fusion at the level of a spondylolisthesis. Right: A myelogram performed 2 years following the decompression and fusion to evaluate radicular symptoms referable to the mid-lumbar level. Stenosis above the previously decompressed and fused level can be seen.

\section{COSTS AND COMPLICATIONS}

The indications for decompressive surgery to treat symptomatic spinal stenosis are well established. However, the associated benefit of concomitant spinal fusion has not been as well defined. The clinical significance of the finding of radiographic instability exhibited after decompressive lumbar surgery remains unclear. Often these radiographic signs of instability are not associated with enough clinical symptoms to warrant the addition of a fusion procedure with the associated costs, complications, and the potential failures.[54,60]

Deyo, et al.,[10] in a review of Medicare patients undergoing lumbar decompression for spinal stenosis, found that those patients who underwent fusion had nearly twice the complication rate of those treated with decompression alone. In addition, the fusion group had nearly six times the transfusion rate and twice the rate of nursing home placement, and a significantly higher postoperative morbidity rate.

In a review of the epidemiological concerns, Katz[36] summarized the surgical rates and associated costs of lumbar fusion surgery in the United States. He found the rates of lumbar fusion had dramatically increased in the United States since the 1970s, and the group of patients diagnosed with lumbar stenosis received the largest increase in surgical procedures. He inferred that the increased rate of surgery in the older spinal stenosis population was a result of a number of factors including the increasing age of the population in the United States; the emergence of noninvasive diagnostic imaging (computerized tomography and magnetic resonance imaging); improvements in the pre- and postoperative medical care for the elderly; and the proliferation of new fusion techniques and spinal instrumentation technology. He also found a marked variation in the rates at which surgeons in different geographical areas performed 
fusions, which, Katz suggested, was indicative of poorly defined criteria for the indications for fusion. The patients undergoing fusion had a significantly higher complication rate, mortality rate, and a higher likelihood of being discharged into a supportive care facility. In addition, there were longer hospital stays and higher hospital costs. The hospital cost of laminectomy with fusion was $50 \%$ higher than with the decompressive laminectomy alone, and when instrumentation was added to the fusion procedure, the cost was $100 \%$ higher than the laminectomy alone. Other authors have found similarly increased complication rates in the instrumentation and fusion group of patients without a significantly improved

outcome.[10,15,18,36,61,62]

In a metaanalysis of the literature on surgery for spinal stenosis, Turner, et al.,[62] found that decompressive lumbar laminectomy for stenosis was a satisfactory treatment with a low complication rate. No improvement in the outcome was found when fusion was combined with lumbar decompression. These findings emphasized the need for a more rigorously controlled study addressing the benefits of spinal fusion when balanced against the associated increased morbidity rates. The increased complications associated with fusion surgery included wound infections, deep vein thromboses/pulmonary embolus, neural injury, graft site complications, and an instrument failure rate of up to $8 \%$ of the cases.

\section{CURRENT AND FUTURE FUSION TECHNIQUES}

Many different operative approaches and techniques have been proposed to enhance the arthrodesis rate in the lumbar spine. The approaches may be anterior, posterior, or posterolateral, and they may also involve a variety of fusion techniques. The most common technique is that of posterolateral intertransverse fusion, which has the widest application and the most versatility when fusing more than one spinal level.[21,44] Posterior midline fusions provide an acceptable alternative when confined to one spinal level.[16,21,44] Anterior interbody fusions [30] or posterior interbody fusions[9] have variable fusion results depending on the technique. Often instrumentation is required to enhance the fusion success and provide some degree of deformity reduction.[6,37,57] New surgical devices involving bone-filled "cages" or "baskets" have been recently developed and are designed to standardize and enhance interbody fusion techniques.

Current posterolateral fusion techniques require a wide dissection of the paraspinous tissue to provide exposure of the transverse processes. The intertransverse technique requires large exposure with substantial trauma to the supporting paraspinous musculature. In addition to disruption of the blood supply to the muscles and soft tissues, the blood supply to the fusion area may be diminished, resulting in a less-than-satisfactory outcome.[44] Recently, a surgical technique has been proposed that involves an extensive unilateral decompression for lumbar stenosis combined with fusion that requires less extensive soft-tissue disruption. The "ipsi-contra" procedure involves a radically unilateral decompression of an ipsilateral symptomatic nerve root with removal of the facet, ligament, pedicle, and disc if necessary. The spinous process is preserved, and the area is undercut, thereby allowing a decompression of the contralateral side. A contralateral bone fusion of the facet lamina and spinous process is achieved using the bone removed during the decompression as graft material. This procedure not only provides an adequate decompression but also avoids the complications associated with the wide intertransverse exposure and with the remote graft donor site.[11]

Recent advances in fiber optics and imaging modalities have spawned the development of minimally invasive surgical techniques. The application of endoscopic techniques to the spine may allow fusion to 
be performed with less resulting morbidity. Stereotactically guided placement of instrumentation will improve the precision and decrease the complication rate. Currently, autologous bone is the most commonly used graft material. In some cases, allograft has been substituted with good results.[47] However, recent advances in molecular biology have led to the development of growth factors that may be used in future spine fusion procedures in conjunction with these minimally invasive techniques.[23,65]

\section{CONCLUSIONS}

The patient populations presenting for decompressive surgery for degenerative spinal stenosis are frequently of advanced age and have a higher incidence of coexisting medical problems that mandate the enlightened and restrained use of spinal fusion. The decision to perform a concomitant fusion at the time of decompressive surgery must be made after fully evaluating the patient's complaints, physical findings, and the pathological correlation with the neuroimaging. Given the fact that the life expectancy of the patient population undergoing surgery for lumbar stenosis may be more than 20 years, surgical failures are expected to accumulate over time, and the surgical treatment cannot be considered absolute. The initial surgical triumphs with fusion and instrumentation may ultimately become diminished by long-term complications related to the implanted devices.

Although myelography or a combination of myelography with computerized tomography scanning provides the most accurate rendering of the degenerative lumbar spine in unstable pathological conditions such as with spondylolisthesis or scoliosis, the magnetic resonance imaging is exerting greater influence and is becoming the preeminent imaging modality. Standing flexion-extension radiographs are essential in the evaluation of stability in the degenerative lumbar spine.

Based on this review and our interpretation of the current status of the literature, we would recommend spinal fusion under the following absolute anatomical conditions. Spinal fusion is indicated in the following situations: 1) A decompressive laminectomy in the presence of segmental instability, as manifested by gross movement on flexion-extension radiographs of greater than $4 \mathrm{~mm}$ of translation or $10 \check{s}$ of angular displacement. 2) A decompressive laminectomy at an area of degenerative instability, as in the case of a coexisting spondylolisthesis or scoliosis where the disc height is maintained. 3) A decompressive laminectomy in a segment with preserved disc heights, where an adequate decompression requires the removal of greater than $50 \%$ of both facets or the complete removal of a unilateral facet complex.

Spinal fusion with segmental fixation is indicated in the following: 1) A decompressive laminectomy at an area of degenerative instability, as in the case of a coexisting spondylolisthesis or scoliosis when a discectomy is performed at the same level. 2) A decompressive laminectomy at an area of degenerative instability, as in the case of a coexisting spondylolisthesis or scoliosis, where there is gross movement on flexion-extension radiographs. 3) A decompressive laminectomy at an area of degenerative instability, as in the case of a coexisting spondylolisthesis or scoliosis, where an adequate decompression requires the removal of greater than $50 \%$ of both facets or the complete removal of a unilateral facet complex.

Concomitant spinal fusion is not indicated in the following: 1) A routine decompressive laminectomy when a discectomy is necessary to provide an adequate decompression or when multiple spinal segments are involved in the decompression. 2) A decompressive laminectomy at an area of degenerative instability, as in the case of a coexisting spondylolisthesis or scoliosis, where there is disc space narrowing and osteophyte formation at the same level, in elderly patients in whom mechanical back pain 
is not the major symptom. 3) Decompressive laminectomy at an area of degenerative instability, as in the case of a coexisting spondylolisthesis or scoliosis, where the decompression was limited to an isolated unilateral segment for radicular symptoms.

The patient's symptomatic complaints and physical findings have been progressively overshadowed by each successive generation of sophisticated imaging technology and are no longer the dominant factor in the surgical decision-making process. From the patient's perspective, the end point is the resolution of pain. A successful surgical outcome must incorporate the patient's expectations. The patient typically complains of either back pain, which is perceived as a "bone" origin, or leg pain, which is perceived as originating from a "nerve." The surgeon is charged with determining whether the patient's complaints are referable to a primary back (bone) disease with the inherent mechanical and stability implications or whether it is a primary neurological (nerve) deficit related to a structural compromise of a nerve root or the cauda equina; or as is more frequently the case, the surgeon must determine the relative contribution the various anomalies make to the patient's complaints. The indications for the various surgical procedures are multifactorial and must involve consideration of the patient's age, coexisting medical problems, and level of activity. Surgical judgment is of paramount importance in determining the appropriate surgical procedure for each individual patient with lumbar stenosis.

\section{References}

1. Abumi K, Panjabi MM, Kramer VM, et al: Biomechanical evaluation of lumbar spine stability after graded facetectomies. Spine 15:1142-1147, 1990

2. Albee FH: Transplantation of a portion of the tibia into the spine for Pott's disease. JAMA 57:885-886, 1911

3. Alexander E Jr, Kelly DL Jr, Davis CH Jr, et al: Intact arch spondylolisthesis. A review of 50 cases and description of surgical treatment. J Neurosurg 63:840-844, 1985

4. Aryanpur J, Ducker T: Multilevel lumbar laminotomies: an alternative to laminectomy in the treatment of lumbar stenosis. Neurosurgery 26:429-433, 1990

5. Bridwell KH, Sedgewick TA, O'Brien MF, et al: The role of fusions and instrumentation in the treatment of degenerative spondylolisthesis with spinal stenosis. J Spinal Disord 6:461-472, 1993

6. Brodsky AE: Post-laminectomy and post-fusion stenosis of the lumbar spine. Clin Orthop 115:130-139, 1976

7. Caputy AJ, Luessenhop AJ: Long-term evaluation of decompressive surgery for degenerative lumbar stenosis. J Neurosurg 77:669-676, 1992

8. Chen Q, Baba H, Kamitani K, et al: Postoperative bone re-growth in lumbar spinal stenosis. A multivariate analysis of 48 patients. Spine 19:2144-2149, 1994

9. Cloward RB: The treatment of ruptured lumbar intervertebral discs by vertebral body fusion: I. Indications, operative technique, after care. J Neurosurg 10:154-168, 1953

10. Deyo RA, Ciol MA, Cherkin DC, et al: Lumbar spinal fusion. A cohort study of complications, reoperations, and resource use in the Medicare population. Spine 18:1463-1470, 1993 
11. diPierro CG, Helm GA, Shaffrey CI, et al: Treatment of lumbar spinal stenosis by extensive unilateral decompression and contralateral autologous bone fusion: operative technique and results. J Neurosurg 84:166-173, 1996

12. Esses SI, Huler RJ: Indications for lumbar spine fusion in the adult. Clin Orthop 279:87-100, 1992 13. Feffer HL, Wiesel SW, Cucker JM, et al: Degenerative spondylolisthesis. To fuse or not to fuse. Spine 10:287-289, 1985

14. Fox MW, Onofrio BM, Hanssen AD: Clinical outcomes and radiological instability following decompressive lumbar laminectomy for degenerative spinal stenosis: a comparison of patients undergoing concomitant arthrodesis versus decompression alone. J Neurosurg 85:793-802, 1996

15. Franklin GM, Haug J, Heyer NJ, et al: Outcomes of lumbar fusion in Washington State workers' compensation. Spine 19:1897-1904, 1994

16. Frymoyer JW: Segmental instability: overview and classification, in Frymoyer JW (ed): The Adult Spine: Principles and Practice. New York: Raven Press, 1991, pp 1873-1891

17. Grabias S: The treatment of spinal stenosis. J Bone Joint Surg (Am) 62:308-313, 1980

18. Grob D, Humke T, Dvorak J: Degenerative lumbar spinal stenosis. Decompression with and without arthrodesis. J Bone Joint Surg (Am) 77:1036-1041, 1995

19. Grubb SA, Lipscomb HJ: Results of lumbosacral fusion for degenerative disc disease with and without instrumentation: two to five year follow up. Spine 17:349-355, 1992

20. Hanley EN Jr: The indications for lumbar spinal fusion with and without instrumentation. Spine 20 (Suppl 24):143S-153S, 1995

21. Hanley EN Jr, Phillips ED, Kostuik JP: Who should be fused?, in Frymoyer JW (ed): The Adult Spine: Principles and Practice. New York: Raven Press, 1991, pp 1893-1917

22. Hazlett JW, Kinnard P: Lumbar apophyseal process excision and spinal stability. Spine 7:171-176, 1982

23. Helm GA, Sheehan JM, Sheehan JP, et al: Utilization of type I collagen gel, demineralized bone matrix, and bone morphogenic protein-2 to enhance autologous bone lumbar spinal fusion. J Neurosurg 86:93-100, 1997

24. Herkowitz HN, Kurz LT: Degenerative lumbar spondylolisthesis with spinal stenosis. A prospective study comparing decompression with decompression and intertransverse process arthrodesis. J Bone Joint Surg (Am) 73:802-808, 1991

25. Herno A, Airaksinen O, Saari T: Long-term results of surgical treatment of lumbar spinal stenosis. Spine 18:1471-1474, 1993

26. Herron LD, Trippi AC: L4-5 degenerative spondylolisthesis. The results of treatment by decompression laminectomy without fusion. Spine 14:534-538, 1989

27. Hibbs RA: The treatment of deformities of the spine caused by poliomyelitis; a report of eight cases in which fusion operations were performed. JAMA 69:787-791, 1917 
28. Hopp E, Tsou PM: Postdecompression lumbar instability. Clin Orthop 227:143-151, 1988

29. Howorth MB: Evolution of spinal fusion. Ann Surg 117:278-289, 1943

30. Inoue S, Watanabe T, Goto S, et al: Degenerative spondylolisthesis. Pathophysiology and results of anterior interbody fusion. Clin Orthop 227:90-98, 1988

31. Johnsson KE, Redlund-Johnell I, Uden A, et al: Preoperative and postoperative instability in lumbar spinal stenosis. Spine 14:591-593, 1989

32. Johnsson KE, Rosen I, Uden A: The natural course of lumbar spinal stenosis. Clin Orthop 279:82-86, 1992

33. Johnsson KE, Willner S, Johnsson K: Postoperative instability after decompression for lumbar stenosis. Spine 11:107-110, 1986

34. Johnsson KE, Willner S, Pettersson H: Analysis of operated cases with lumbar spinal stenosis. Acta Orthop Scand 52:427-433, 1981

35. Kaneda K, Kazama H, Satoh S, et al: Follow-up study of medial facetectomies and posterolateral fusion with instrumentation in unstable degenerative spondylolisthesis. Clin Orthop 203:159-167, 1986

36. Katz JN: Lumbar spinal fusion. Surgical rates, costs, and complications. Spine 20 (Suppl 24):78S-83S, 1995

37. Kostuik JP: Recent advances in the treatment of painful adult scoliosis. Clin Orthop 147:238-252, 1980

38. Kostuik JP, Errico TJ, Gleason TF: Techniques of internal fixation for degenerative conditions of the lumbar spine. Clin Orthop 203:219-231, 1986

39. Lang F: Support for the spondylitic spine by means of buried steel bars attached to the vertebrae. Am J Orthop Surg 8:344, 1910

40. Lee CK: Lumbar instability (olisthesis) after extensive posterior spinal decompression. Spine 8:429-433, 1983

41. Lehmann TR, Spratt KF, Tozzi JE, et al: Long-term follow-up of lower lumbar fusion patients. Spine 12:97-104, 1987

42. Lehto MUK, Honkanen P: Factors influencing the outcome of operative treatment for lumbar spinal stenosis. Acta Neurochir 137:25-28, 1995

43. Lombardi JS, Wiltse LL, Reynolds J, et al: Treatment of degenerative spondylolisthesis. Spine 10:821-827, 1985

44. Macnab I, Dall D: The blood supply of the lumbar spine and its application to the technique of intertransverse lumbar fusion. J Bone Joint Surg (Br) 53:629-638, 1971

45. Mardjetko SM, Connolly PJ, Shott S: Degenerative lumbar spondylolisthesis. A meta-analysis of the literature 1970-1993. Spine 19(Suppl 20):2256S-2265S, 1994 
46. Nachemson A: Adult scoliosis and back pain. Spine 4:513-517, 1979

47. Nasca RJ: Rationale for spinal fusion in lumbar spinal stenosis. Spine 14:451-454, 1989

48. Osterman K, Lindholm TS, Laurent LE: Late results of removal of the loose posterior elements (Gill's operation) in the treatment of lytic lumbar spondylolisthesis. Clin Orthop 117:121-128, 1976

49. Paine KW: Results of decompression for lumbar spinal stenosis. Clin Orthop 115:96-100, 1976

50. Postacchini F, Cinotti G: Bone regrowth after surgical decompression for lumbar spinal stenosis. J Bone Joint Surg (Br) 74:862-869, 1992

51. Reynolds JB, Wiltse LL: Surgical treatment of degenerative spondylolisthesis. Spine 4:148-149, 1979 (Abstract)

52. San Martino A, D'Andria FM, San Martino C: The surgical treatment of nerve root compression caused by scoliosis of the lumbar spine. Spine 8:261-265, 1983

53. Saraste H: Spondylolysis and spondylolisthesis. Acta Orthop Scand Suppl 251:84-86, 1993

54. Silvers HR, Lewis PJ, Asch HL: Decompressive lumbar laminectomy for spinal stenosis. J Neurosurg 78:695-701, 1993

55. Simmons ED Jr, Simmons EH: Spinal stenosis with scoliosis. Spine 17 (Suppl):S117-S120, 1992

56. Sonntag VKH, Marciano FF: Is fusion indicated for lumbar spine disorders? Spine 20 (Suppl 24):138S-142S, 1995

57. Steffee AD, Sitkowski DJ: Posterior lumbar interbody fusion and plates. Clin Orthop 227:99-102, 1988

58. Strömqvist B: Postlaminectomy problems with reference to spinal fusion. Acta Orthop Scand Suppl 251:87-89, 1993

59. Surin V, Hedelin E, Smith L: Degenerative lumbar spinal stenosis: results of operative treatment. Acta Orthop Scand 53:79-85, 1982

60. Sypert GW: Low back pain disorders: lumbar fusion? Clin Neurosurg 33:457-483, 1986

61. Tuite GF, Stern JD, Doran SE, et al: Outcome after laminectomy for lumbar spinal stenosis. Part I: Clinical correlations. J Neurosurg 81:699-706, 1994

62. Turner JA, Erpsek M, Herron L, et al: Surgery for lumbar spinal stenosis. Attempted meta-analysis of the literature. Spine 17:1-8, 1992

63. Verbiest H: Results of surgical treatment of idiopathic developmental stenosis of the lumbar vertebral canal. A review of twenty-seven years' experience. J Bone Joint Surg (Br) 59:181-188, 1977

64. Wiltse LL, Kirkaldy-Willis WH, McIvor GW: The treatment of spinal stenosis. Clin Orthop 115:83-91, 1976

65. Wozney JM, Rosen V, Celeste AJ, et al: Novel regulators of bone formation: molecular clones and activities. Science 242:1528-1534, 1988 
66. Yong-Hing K, Kirkaldy-Willis WH: The pathophysiology of degenerative disease of the lumbar spine. Orthop Clin North Am 14:491-504, 1983

67. Yuan HA, Garfin SR, Dickman CA, et al: A historical cohort study of pedicle screw fixation in thoracic, lumbar and sacral spinal fusions. Spine 19 (Suppl 20):2279S-2296S, 1994

68. Zdebleck TA: The treatment of degenerative lumbar disorders. A critical review of the literature. Spine 20 (Suppl):126S-137S, 1995

Manuscript received June 3, 1997.

Accepted in final form July 15, 1997.

Address reprint requests to: Anthony J. Caputy, M.D., Department of Neurological Surgery, The George Washington University Medical Center, 2150 Pennsylvania Avenue NW, Suite 7-420, Washington, DC 20037. 